\title{
Neutrophil extracellular traps and neutrophil-derived mediators as possible biomarkers in bronchial asthma
}

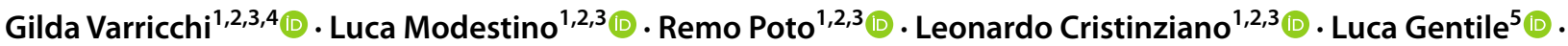 \\ Loredana Postiglione $\mathrm{e}^{1,2,3}$ (-) Giuseppe Spadaro ${ }^{1,2,3}$ (1) $\cdot$ Maria Rosaria Galdiero ${ }^{1,2,3,4}$ (1)
}

Received: 21 June 2021 / Accepted: 25 July 2021 / Published online: 3 August 2021

(c) The Author(s) 2021

\begin{abstract}
Neutrophils (PMNs) contain and release a powerful arsenal of mediators, including several granular enzymes, reactive oxygen species (ROS) and neutrophil extracellular traps (NETs). Although airway neutrophilia is associated with severity, poor response to glucocorticoids and exacerbations, the pathophysiological role of neutrophils in asthma remains poorly understood. Twenty-four patients with asthma and 22 healthy controls (HCs) were prospectively recruited. Highly purified peripheral blood neutrophils ( $>99 \%$ ) were evaluated for ROS production and activation status upon stimulation with lipopolysaccharide (LPS), N-formylmethionyl-leucyl-phenylalanine (fMLP) and phorbol 12-myristate 13-acetate (PMA). Plasma levels of myeloperoxidase (MPO), CXCL8, matrix metalloproteinase-9 (MMP-9), granulocyte-monocyte colony-stimulating factor (GM-CSF) and vascular endothelial growth factor (VEGF-A) were measured by ELISA. Plasma concentrations of citrullinated histone $\mathrm{H} 3$ ( $\mathrm{CitH} 3$ ) and circulating free DNA (dsDNA) were evaluated as NET biomarkers. Activated PMNs from asthmatics displayed reduced ROS production and activation status compared to HCs. Plasma levels of MPO, MMP-9 and CXCL8 were increased in asthmatics compared to HCs. CitH3 and dsDNA plasma levels were increased in asthmatics compared to controls and the $\mathrm{CitH} 3$ concentrations were inversely correlated to the $\%$ decrease in $\mathrm{FEV}_{1} / \mathrm{FVC}$ in asthmatics. These findings indicate that neutrophils and their mediators could have an active role in asthma pathophysiology.
\end{abstract}

Keywords Asthma $\cdot$ Myeloperoxidase $\cdot$ Neutrophil extracellular traps $\cdot$ ROS

Co-first authors: Gilda Varricchi, Luca Modestino, Remo Poto.

Maria Rosaria Galdiero

mariarosaria.galdiero@unina.it

1 Department of Translational Medical Sciences, University of Naples Federico II, 80131 Naples, Italy

2 Center for Basic and Clinical Immunology Research (CISI), University of Naples Federico II, 80131 Naples, Italy

3 World Allergy Organization (WAO) Center of Excellence, 80131 Naples, Italy

4 Institute of Experimental Endocrinology and Oncology (IEOS), National Research Council, 80131 Naples, Italy

5 Department of Molecular Medicine and Medical Biotechnologies, University of Naples Federico II, 80131 Naples, Italy

\section{Introduction}

Bronchial asthma is a heterogeneous chronic inflammatory disease with a broad spectrum of severity [1]. More than 300 million people globally suffer from asthma with up to $10 \%$ of all patients having severe asthma [2,3]. Asthma heterogeneity mirrors the underlying molecular mechanisms and contributes to the variable presentation of the disease [4]. Therefore, asthma is not a single disease and several endotypes/phenotypes of asthma have currently been defined $[5,6]$. According to the type of inflammation, asthma has been divided into $\mathrm{T}$ (type) 2-high and T2-low [7]. T2-high asthma is the most frequent endo/phenotype and is canonically marked by activation of type 2 helper (Th2) cells, type 2 innate lymphoid cells (ILC2s), mast cells, basophils, eosinophils and the production of type 2 cytokines (e.g., IL-3, IL-4, IL-5 and IL-13) [8-10]. Besides $\mathrm{T}_{\mathrm{H}} 2$ cells, also T follicular helper $\left(\mathrm{T}_{\mathrm{FH}}\right)$ cells, a specialized subset of CXCR5 ${ }^{+}$ $\mathrm{CD}^{+}{ }^{+} \mathrm{T}$ cells, are a major source of IL-4 and IL-21 [11], thus closely regulating IgE isotype switching during asthma 
in both humans and mice [12]. T2-high asthma biomarkers are represented by exhaled nitric oxide (FeNO), serum IgE, blood and sputum eosinophils [13]. In T2-low asthma, respiratory viruses, bacteria, fungi and cigarette smoke can induce the release of epithelium-derived alarmin [i.e., IL-33, thymic stromal lymphopoietin (TSLP) and IL-25], which activate a variety of cells of the innate and adaptive immune system [8, 14]. T2-low asthma is characterized by neutrophilic or paucigranulocytic inflammation and the activation of Th1 and/or Th17 cells, ILC1s, ILC3s and the release of specific cytokines (e.g., CXCL8, IL-17) [9, 15]. Specific biomarkers phenotyping T2-low asthma are currently lacking and represent unmet needs. Therefore, the T2-low endotype is a diagnosis of exclusion defined by the absence of $\mathrm{T} 2$ biomarkers [16]. Both neutrophils and CXCL8 have been demonstrated in sputum and blood of severe asthma patients $[17,18]$. CXCL8 is overexpressed in airway secretions of severe asthma patients, and neutrophil airway infiltration is found in severe asthma [19].

Neutrophils (or polymorphonuclear leukocytes: PMNs) are the most abundant circulating leukocytes in humans [20] and are an important component of the host response against pathogens [21-23]. They represent the first line of defense of the innate immune system against infectious agents [24]. During infections, neutrophils migrate from peripheral blood to tissues in response to chemotactic stimuli (e.g., formylated peptides, CXCL8, lipopolysaccharide) released during the inflammatory response [25-27]. PMNs rapidly bind and kill microbes through the release of a potent antimicrobial arsenal, including several granular enzymes [myeloperoxidase (MPO), matrix metalloproteinases (MMPs)] and reactive oxygen species (ROS) [28, 29]. A variety of immunologic and non-immunologic stimuli, including several bacteria [25], fungi [30] and viruses [31, 32] activate human neutrophils to release neutrophilic extracellular traps (NETs), composed by a DNA scaffold with associated granule proteins in a process sometimes called NETosis [33]. NETs bind and kill bacteria [25]. Beyond infectious diseases [25, $34-36]$, NETs play a role in autoimmune disorders $[37,38]$ and cancer [24, 39-41]. There is preliminary evidence that NETs can contribute to some aspects of allergic disorders through different mechanisms [42-47].

The pathophysiological role of neutrophils in asthma has recently begun to emerge [48]. Circulating PMNs from aspirin-exacerbated asthma patients displayed increased ROS production, CD11b expression and CXCL8 and MMP-9 release compared to aspirin-tolerant asthma patients [49]. In patients with allergic asthma, allergen provocation caused a shift in the neutrophil subsets from $\mathrm{CD} 16^{\text {high }} \mathrm{CD} 62 \mathrm{~L}^{\text {high }}$ to $\mathrm{CD} 16^{\text {high }} \mathrm{CD} 62 \mathrm{~L}^{\text {low }}$ [50]. NETs were found in bronchial biopsies of asthmatic patients [47]. In addition, NETs promoted CXCL8 production from human airway epithelial cells [43]. Activated neutrophils release ROS, which contributes to the pathogenesis of asthma by inducing inflammatory injury in airway epithelial cells [51, 52].

In this study, we evaluated the production of ROS from highly purified peripheral blood neutrophils from asthma patients of different severity compared to healthy controls. In addition, we compared the ex vivo activation status of neutrophils, the circulating levels of several neutrophil-derived mediators and of NET-derived biomarkers in healthy donors and asthma patients.

\section{Materials and methods}

\section{Patients and methods}

We enrolled 24 asthma patients (17 females, mean age $50.71 \pm 15$ years and 22 healthy matched donors (17 females, mean age $49.1 \pm 15$ years) from April 2020 to May 2021 at the Division of Clinical Immunology, Allergy and Immunodeficiency of the Department of Translational Medical Sciences, University of Naples Federico II. The study was conducted in accordance with Good Clinical Practice (GCP) guidelines and adhered to the Declaration of Helsinki II. Written informed consent was obtained from all participants. Patients were eligible for enrollment in the study if they were aged 18-70 years and had a history of allergist-diagnosed asthma. Key exclusion criteria were autoimmune diseases, infections, malignancies, a patientreported smoking history or the onset of respiratory symptoms after the age of 40 years in current or previous smokers with a smoking history of at least 10 pack-years. None of the asthmatic patients has been or was treated with allergen-specific immunotherapy [53] or monoclonal antibodies anti-IgE, anti-IL-5/IL-5R $\alpha$ or anti-IL-4R $\alpha$ [4, 54, 55]. The majority of patients (20/24) were treated with daily low-dose of inhaled glucocorticoids (ICS) therapy [fluticasone propionate (FP), 100-200 $\mu \mathrm{g}$ or equivalent] plus two additional controllers (e.g., a long-acting $\beta_{2}$-agonist and/or leukotrienereceptor antagonist); $4 / 24$ of patients were treated with daily medium-dose of ICS (FP, 250-500 $\mu$ g or equivalent); 3/24 of patients were on oral glucocorticoid therapy (mean prednisone intake $14.2 \mathrm{mg} / \mathrm{die}$ ). Baseline characteristics of the patients and healthy volunteers included in the study are shown in Tables 1 and 2. Peripheral blood samples were collected at the Center for Basic and Clinical Immunology Research (CISI), University of Naples Federico II and were immediately processed. Plasma samples were also obtained $\left(+4{ }^{\circ} \mathrm{C}, 400 \mathrm{~g}, 20 \mathrm{~min}\right)$ and stored $\left(-80^{\circ} \mathrm{C}\right)$ until used. The following parameters were evaluated: the on-treatment forced expiratory volume in $1 \mathrm{~s}\left(\mathrm{FEV}_{1}\right.$, in liters); the fraction of exhaled nitric oxide $\left(\mathrm{F}_{\mathrm{ENO}}\right.$, in parts per billion: $\left.\mathrm{ppb}\right)$; the score on the Asthma Control Test (ACT; the mean score of five questions that assess asthma symptoms, use of rescue 
Table 1 Demographic data, clinical characteristics and laboratory data of asthma patients and healthy controls

\begin{tabular}{lll}
\hline Characteristics & Asthma Patients & Healthy Controls \\
\hline Age-years & $50.7 \pm 15$ & $49.1 \pm 15$ \\
Female sex (\%) & 71 & 77 \\
BMI $\left(\mathrm{Kg} / \mathrm{m}^{2}\right)$ & $27.17 \pm 5$ & $27.51 \pm 4.2$ \\
Number of hospital admissions for asthma at any time & $0.5 \pm 0.9$ & None \\
$\quad$ before enrollment & & \\
ACT score & $14.5 \pm 5.3$ & NA \\
Patients with concomitant nasal polyposis $(\%)$ & 41.7 & None \\
FEV $(\%$ of predicted value) & $69.7 \pm 4.2^{* * * * *}$ & $103 \pm 11$ \\
$\mathrm{~F}_{\text {ENO }(\mathrm{ppb})}$ & $48.4 \pm 23^{* * * *}$ & $8.1 \pm 3$ \\
Blood neutrophil count $\left(\right.$ cells $\left./ \mathrm{mm}^{3}\right)$ & $4,160 \pm 401 * * *$ & $3,371 \pm 955$ \\
Blood eosinophil count $\left(\right.$ cells $\left./ \mathrm{mm}^{3}\right)$ & $384 \pm 18^{* * * *}$ & $130 \pm 78$ \\
Total IgE $(\mathrm{IU} / \mathrm{ml})$ & $264.3 \pm 70^{* * * *}$ & $69.2 \pm 107$ \\
\hline
\end{tabular}

NA: not applicable; $* p<0.05 ; * * p<0.01 ; * * * p<0.0005 ; * * * * p<0.0001$ medications and the effect of asthma on daily functioning during the previous 4 weeks) [56]. Spirometry (Quark PTF, COSMED, Pavona di Albano, Italy) (before and after salbutamol $400 \mathrm{mcg}$ ) was performed in accordance with the American Thoracic Society/European Respiratory Society (ATS/ERS) guidelines [57]. FEV ${ }_{1}$, Forced Vital Capacity (FVC), $\mathrm{FEV}_{1} / \mathrm{FVC}$ were measured and the best of three forced maneuvers was recorded. Results were expressed both as absolute values and as a percentage of the predicted values referred to European Respiratory Society (ERS) reference values [58]. FeNO was measured using an electrochemical analyzer (HypairFeNOMedisoftExp'air, Dinant, Belgium) according to ATS/ERS recommendations for online measurement of exhaled NO in adults [59]. Peripheral blood leukocyte counts were measured using an automated hematology analyzer.

\section{Neutrophil purification and culture}

The study protocol, involving the use of human blood cells was approved by the Ethics Committee of the University of Naples Federico II (198/18), and written informed consent was obtained from blood donors according to the principles expressed in the Declaration of Helsinki. Leukocytes from peripheral blood of asthma patients and healthy controls were separated from erythrocytes by dextran sedimentation. Neutrophils were purified by Ficoll-Paque Histopaque ${ }^{\circledR}-1077$ (Sigma-Aldrich, Milan, Italy) density gradient centrifugation $\left(400 \times \mathrm{g}, 30 \mathrm{~min},+22^{\circ} \mathrm{C}\right)$, followed by Percoll (Sigma-Aldrich, Milan, Italy) $(65 \%)$ density gradient centrifugation $\left(660 \times g, 20 \mathrm{~min},+22{ }^{\circ} \mathrm{C}\right)$ as previously described [60]. Neutrophils were isolated from granulocytes (to reach $>99 \%$ purity) by positive elimination of all contaminating cells using the EasySep Neutrophil Enrichment Kit (StemCell Technologies, Vancouver, Canada) [61]. These cells were $>99 \%$ neutrophils as evaluated by flow cytometric analysis with the following antibodies: anti-CD3, anti-CD14, anti-CD15, anti-CD11b, anti-CD193 (Miltenyi Biotec, Bergisch Gladbach, Germany), anti-CD62L (L-selectin) (BD Biosciences, San Jose, CA, USA) and antiCD66b (BioLegend, CA, USA). Samples were analyzed on the MACSQuant Analyzer 10 (Miltenyi Biotec, Bergisch Gladbach, Germany) and in the FlowJo software, v.10. Dead cells, doublets, debris and eosinophils were excluded from the analysis. Data were expressed as percentage of positive cells or median fluorescence intensity [62].

\section{Reactive oxygen species (ROS) production}

Neutrophils $\left(2 \times 10^{6}\right.$ cells $\left./ \mathrm{mL}\right)$ were resuspended in the RPMI 1640 medium with 5\% of fetal bovine serum (FBS) (Euroclone, Milan, Italy) and antibiotics at $37{ }^{\circ} \mathrm{C}$ and $5 \%$ $\mathrm{CO}_{2}$. The cells were incubated for $30 \mathrm{~min}$ after the addition of $\mathrm{H}_{2}$ DCF-DA $10 \mu \mathrm{g} / \mathrm{mL}$ (Life Technologies, Milan Italy). $\mathrm{H}_{2}$ DCF-DA is a fluorogenic dye that allows to determine hydroxyl peroxyl and other ROS activities within the cell [60]. Once diffused into the cell, $\mathrm{H}_{2}$ DCF-DA is deacetylated by cellular esterases to a non-fluorescent molecule, which is oxidized by ROS into 2',7' dichlorofluorescein (DCF). DCF, a highly fluorescent molecule, is oxidized by fluorescent spectroscopy with maximum excitation and emission wavelengths of 492-495 and 517-527 nm, respectively. Neutrophils were washed in PBS and resuspended in RPMI 5\% FBS in the presence or absence of different immunologic (LPS; $100 \mathrm{ng} / \mathrm{ml}$ ) or fMLP; $1 \mu \mathrm{M}$ ) or non-immunologic stimuli (phorbol 12-myristate 13-acetate: PMA; $10 \mathrm{ng} / \mathrm{ml}$ ) (SigmaAldrich, Milan, Italy) and immediately analyzed in a 96-well plate and placed in a EnSpire Multimode Plate Reader (Perkin Elmer, Waltham, MA, USA). DCF mean fluorescence intensity was measured at an excitation wavelength of $492-495 \mathrm{~nm}$ and emission at $517-527 \mathrm{~nm}$. The ability of LPS and fMLP to induce cytoplasmic ROS-catalyzed 


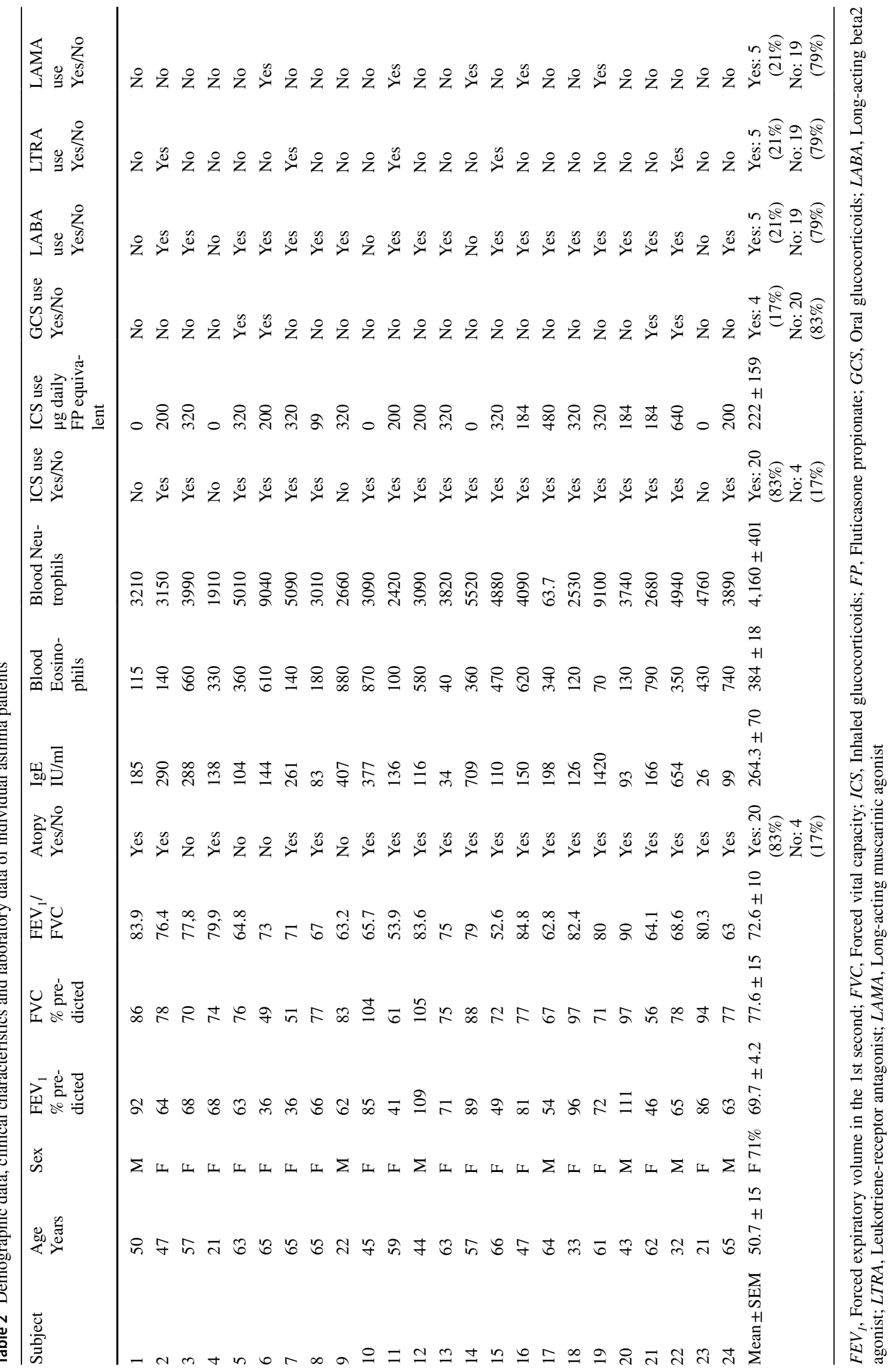


oxidation of DCFH in neutrophils was measured as compared to negative control.

\section{Flow cytometry}

Highly purified (>99\%) neutrophils were kept in RPMI with $10 \%$ of FCS for $30 \mathrm{~min}$ at $+37{ }^{\circ} \mathrm{C}$, then were washed with PBS solution and stimulated with LPS $(100 \mathrm{ng} / \mathrm{ml})$, fMLP $(1 \mu \mathrm{M})$ or PMA $(10 \mathrm{ng} / \mathrm{ml})$. Cells maintained in RPMI 1640 medium containing 5\% of FBS (Euroclone, Milan, Italy) were the negative control $\left(60 \mathrm{~min},+37^{\circ} \mathrm{C}\right)$. $2 \times 10^{5}$ cells were seeded in 96-well plate (Thermo Fisher Scientific, Waltham, MA, USA) and Zombie Violet dye (BioLegend, CA, USA) was added to evaluate cell viability $\left(20 \mathrm{~min},+4{ }^{\circ} \mathrm{C}\right)$. Then, the cells were stained $\left(20 \mathrm{~min},+4{ }^{\circ} \mathrm{C}\right)$ in PBS containing $1 \%$ FBS. The following antibodies were used: allophycocyanin (APC)-conjugated anti-CD66b (clone REA306, dilution 1:50, from Miltenyi Biotech, Bergisch Gladbach, Germany), peridinin chlorophyll protein (PerCP)-conjugated anti-CD11b (clone REA713, dilution 1:50, from Miltenyi Biotech, Bergisch Gladbach, Germany), fluorescein isothiocyanate (FITC)conjugated anti-CD62L (clone 145/15, dilution 1:50, from Miltenyi Biotech, Bergisch Gladbach, Germany) and Phycoerythrin (PE)-conjugated anti-CD16 (clone REA423, dilution 1:50, from Miltenyi Biotech, Bergisch Gladbach, Germany). Cells were acquired by MACS Quant Analyzer 10 (Miltenyi Biotec, Bergisch Gladbach, Germany) and analyzed by FlowJo v.10 software. Doublets and debris (identified based on forward and side scatter properties), dead cells (identified with Zombie Violet Fixable Viability Kit; BioLegend) and eosinophils (identified based on the $\mathrm{CCR} 3^{+}$exclusion gate) were excluded from the analysis. A representative example of the complete gating strategy is illustrated in Supplementary Fig. 1. Data are expressed as a percentage of positive cells or median fluorescence intensity [62].

\section{Measurement of neutrophil-related mediators}

Plasma levels of myeloperoxidase (MPO), matrix metalloproteinase 9 (MMP-9), CXCL8/IL-8, granulocyte-monocyte colony-stimulating factor (GM-CSF) and vascular endothelial growth factor (VEGF-A) of asthma patients and healthy controls were measured using ELISA kits according to the manufacturer's instructions (DuoSet ELISA kit, R\&D Systems, Minneapolis, MN, USA). A microplate reader (Tecan, Grodig, Austria, $\mathrm{GmbH}$ ) was used to determine sample absorbance at $450 \mathrm{~nm}$. The ELISA detection range was $62.5-4000 \mathrm{pg} / \mathrm{ml}$ (MPO), 31.2-2000 pg/ml (MMP-9), 31.2-2000 pg/ml (CXCL8/IL-8), 7.80-500 pg/ml (GMCSF) and 31-2000 pg/ml (VEGF-A).

\section{Plasma NET detection}

Plasma circulating free DNA (dsDNA), a marker for NET formation [63, 64], was measured using a Quant-iT ${ }^{\mathrm{TM}}$ PicoGreen dsDNA Assay Kit (Thermo Fisher Scientific, Waltham, MA, USA) as described in the manufacturer's instructions. Concentration of Citrullinated Histone H3 (CitH3) in plasma samples of asthma patients and healthy controls was measured using ELISA kit developed by Cayman Chemicals (Ann Arbor, MI, USA) according to the manufacturer's instructions. This assay used a specific monoclonal antibody for histone $\mathrm{H} 3$ citrullinated at residue R2, R8 and R17 (clone 11D3). A microplate reader (Tecan, Grodig, Austria, $\mathrm{GmbH}$ ) was used to determine sample absorbance at $450 \mathrm{~nm}$. The ELISA sensitivity is $0.15-10 \mathrm{ng} /$ $\mathrm{ml}$. The H3Cit measurement was set to $0 \mathrm{ng} / \mathrm{ml}$ when the absorbance of a patient sample was lower than the buffer blank. All measurements were executed in duplicates and results are expressed as $\mathrm{ng} / \mathrm{ml}$.

\section{Statistical analysis}

Statistical analysis was performed by using Prism 7 (GraphPad software). Data are expressed as mean \pm SEM of the indicated number of experiments. D'Agostino \& Pearson normality test was used to test for normality of distribution, and statistical methods were chosen to fit non-normal distributions when appropriate. Values from groups were compared by Student's t test or Mann-Whitney U test based on the parametric or nonparametric distribution of the continuous variables. Repeated measures one-way or two-way ANOVA was used were appropriated and described in the figure legends. Differences were assumed to be statistically significant when $p<0.05$.

\section{Results}

\section{Clinical characteristics of the study subjects}

In this study, we enrolled 24 asthma patients and 22 healthy matched controls. Table 1 summarizes their clinical characteristics. The mean age of asthmatic patients was $50.7 \pm 15$ years and $71 \%$ were female. Asthma patients had $0.5 \pm 0.9$ hospital admissions for asthma exacerbation at any time before enrollment. The ACT score was $14.5 \pm 5.3$ and $41.7 \%$ of patients had concomitant nasal polyposis. The $\%$ of predicted value of $\mathrm{FEV}_{1}$ was significantly lower in asthmatics compared to healthy donors $(69.7 \pm 4.2 \%$ vs. $103 \pm 11 \%$; $\mathrm{p}<0.0001$ ). The median $\mathrm{F}_{\mathrm{ENO}}$ was $48.4 \pm 23 \mathrm{ppb}$. The absolute neutrophils count in asthma patients was increased than normal subjects $(4,160 \pm 401$ vs. $3,371 \pm 955 ; \mathrm{p}<0.0005)$. The absolute eosinophil count was significantly higher 
in asthmatics compared to healthy subjects $(384 \pm 18 v s$. $130 \pm 78 ; p<0.0001)$. Serum IgE levels were $264.3 \pm 70 \mathrm{IU} /$ $\mathrm{ml}$ in asthmatic patients and $69.2 \pm 107 \mathrm{IU} / \mathrm{ml}$ in control subjects $(p<0.0001)$. Demographics, clinical characteristics and laboratory data of individual asthma patients are presented in Table 2 . Twenty patients (83\%) were atopic; 20 patients $(83 \%)$ were treated with inhaled glucocorticoids (ICS) and four patients (17\%) were treated with oral glucocorticoids (OCS). None of the patients has been or was treated with allergen-specific immunotherapy or monoclonal antibodies anti-IgE, anti-IL-5/IL-5R $\alpha$ or anti-IL-4R $\alpha$.

\section{ROS production by human neutrophils}

ROS are key signaling molecules that play important roles in the progression of several inflammatory disorders [65], including certain forms of asthma [49]. Highly purified neutrophils (> 90\%) from asthma patients and healthy controls were activated by exposure to LPS, which activates toll-like receptor 4 (TLR4) [66] and to fLMP, which activates formyl peptide receptors [27]. Cells were also activated by PMA, a protein kinase $\mathrm{C}$ (PKC) agonist [25]. To test whether neutrophil activation leads to ROS production in asthma patients, we performed a $2^{\prime}, 7^{\prime}$-dichlorodihydrofluorescein diacetate $\left(\mathrm{H}_{2} \mathrm{DCF}-\mathrm{DA}\right) \mathrm{ROS}$ detection assay. Figure 1 illustrates the kinetics (2 to $30 \mathrm{~min}$ ) of the production of ROS induced by LPS (Fig. 1A), fMLP (Fig. 1B) and PMA (Fig. 1C). ROS production by PMNs was significantly increased by all the inflammatory stimuli in asthma patients as well as in healthy controls. ROS production induced by LPS progressively increased from 2 to $30 \mathrm{~min}$ in normal PMNs. The production of ROS from PMNs isolated from asthma patients had a lag period of approximately $15 \mathrm{~min}$ and then progressively increased. PMNs purified from asthma patients increased ROS production but to a lower extent compared to neutrophils obtained from healthy donors. Figure 1A shows that the difference in LPS-induced ROS production in PMNs from asthma patients and healthy controls was evident from $6 \mathrm{~min}$ onward after stimulation. The shape of kinetics of fMLPinduced ROS production from controls was different from that caused by LPS. There was a rapid increase that tended to plateau after 30 min of incubation. fMLP-induced ROS production was stronger compared to LPS-induced ROS production but the difference between patients and controls was evident starting from 10 min onward after stimulation (Fig. 1B). PMA, a non-immunologic PKC activator, induced ROS production from neutrophils. Figure $1 \mathrm{C}$ shows that also the kinetics of PMA-induced ROS production from asthma patients and from healthy controls were different from those induced by LPS and fMLP. There was a slow increase that did not plateau after 30 min of incubation. ROS production was significantly higher in healthy subjects compared to asthmatics from 20 min onward after PMA stimulation. No difference in ROS production was evident between patients and controls when medium alone was used as negative control (data not shown). Collectively, these findings suggested that ROS production from asthma patient PMNs was lower compared with healthy controls when cells were activated by immunologic and non-immunologic stimuli.

\section{Neutrophil activation in asthma patients}

To determine the activation status of human PMNs isolated from peripheral blood of asthma patients, we determined CD1 1b, CD16 and CD62L (L-selectin) expression on PMNs by flow cytometry $[67,68]$. PMNs stimulated with PMA (as a positive control), fMLP, LPS or control medium, were stained with antibodies against CD11b, CD16 and CD62L and evaluated by flow cytometry. [69]. Under basal conditions, neutrophils from controls and asthmatics showed minimal expression of $\mathrm{CD} 11 \mathrm{~b}$, which rapidly increased after incubation with all inflammatory stimuli tested. PMNs
A

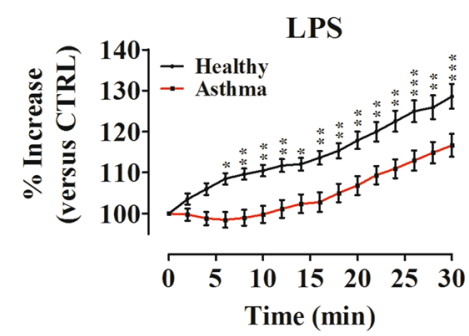

Fig. 1 Neutrophils purified from peripheral blood of asthma patients (red lines) and healthy controls (black lines) were incubated (30 min, $37{ }^{\circ} \mathrm{C}$ ) with $2^{\prime}, 7^{\prime}$-dichlorodihydrofluorescein diacetate $\left(\mathrm{H}_{2} \mathrm{DCFDA}\right.$, $10 \mu \mathrm{M})$, washed and then stimulated with LPS $(100 \mathrm{ng} / \mathrm{mL})(\mathbf{A})$, fMLP $(1 \mu \mathrm{M})(\mathbf{B})$ or PMA $(10 \mathrm{ng} / \mathrm{mL})(\mathbf{C})$. Immediately after stimulation, PMNs were analyzed in a multimode microplate reader
B

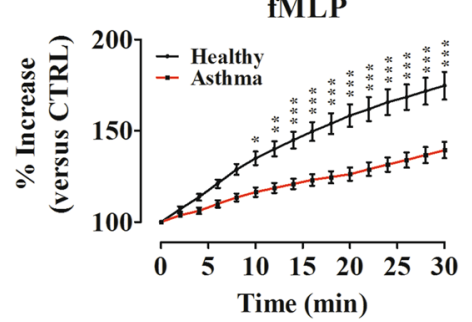

(EnSpire Multimode Plate reader, PerkinElmer) and DCF fluorescence was measured for $30 \mathrm{~min}$ at $2 \mathrm{~min}$ intervals. The results were expressed as percentage increase versus time 0 (mean $\pm \mathrm{SEM}$ ); $* p<0.05 ; * * p<0.01 ; * * * p<0.005$. Two-way ANOVA and Bonferroni post-test 
purified from asthma patients increased CD11b expression to a lower extent compared with healthy controls under LPS (Fig. 2A), fMLP (Fig. 2B) and PMA (Fig. 2C) stimulation. Figure 2D-F shows representative histograms illustrating CD11b expression on peripheral blood neutrophils from healthy controls (HC) and asthma patients (Pt), non-stimulated (CTRL) or stimulated with LPS (D), fMLP (E) and PMA (F).

Under resting conditions, neutrophils expressed CD62L, which rapidly decreased (i.e., shedding) after stimulation with the three examined stimuli. Indeed, the CD16 $6^{\text {bright/ }}$ CD62 $\mathrm{L}^{\mathrm{dim}}$ cells consist mainly of neutrophils containing hyper-segmented nuclei that show a more activated state [70]. Interestingly, under LPS, fMLP or PMA stimulation, PMNs purified from asthma patients underwent CD62L shedding, but to a lesser extent compared to healthy donors (Fig. 3A-C). Figure 3D-K shows representative flow cytometric panels with respect to the gating strategy and $\mathrm{CD} 16^{+} \mathrm{CD} 62 \mathrm{~L}^{-}$cell counts for healthy controls $(\mathrm{D}-\mathrm{G})$ and asthma patients (H-K), stimulated with LPS (E, I), fMLP (F, $\mathrm{J})$ and PMA (G, K) or control medium (D, H). Collectively, these data indicate that when challenged with inflammatory stimuli, peripheral blood PMNs from asthmatics displayed a reduced activation status $(\mathrm{CD} 11 \mathrm{~b}$ up-regulation and CD62L shedding) compared to PMNs purified from controls.

\section{Plasma levels of neutrophil-derived mediators}

Human neutrophils contain and release of a variety of proinflammatory mediators [28, 71]. Myeloperoxidase (MPO), contained in neutrophil primary/azurophilic granules, plays a pivotal role in ROS production [72, 73] and NET formation $[74,75]$. Matrix metalloproteinase-9 (MMP-9) plays a key role in the development of airway inflammation [76-79] and facilitates angiogenesis in asthma [80]. Circulating [81] and BAL levels of CXCL8 [82] are increased in asthmatics. GM-CSF is considered a key orchestrator of neutrophil involvement in chronic airway inflammation [83, 84].
A
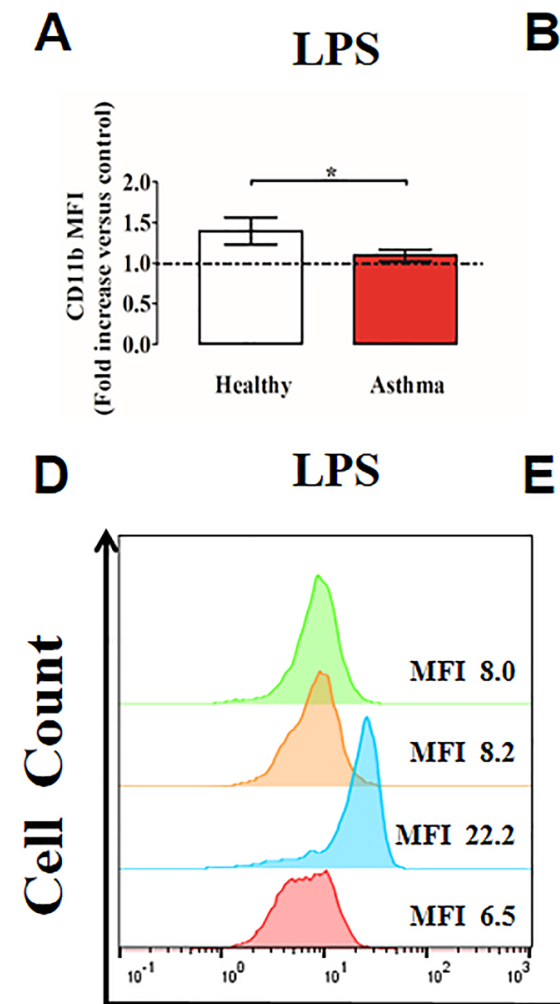

B

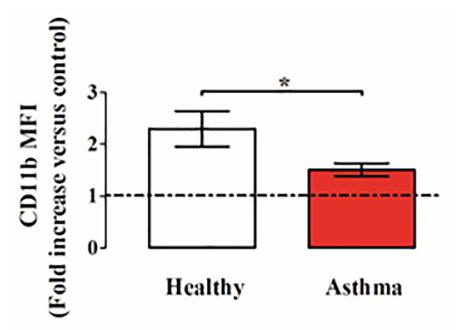

E

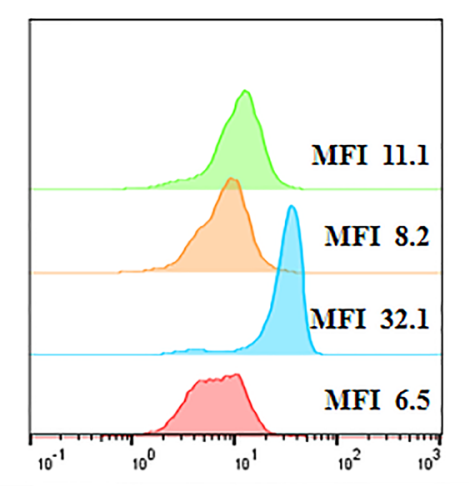

C

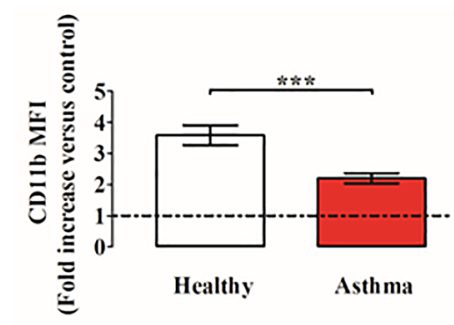

PMA

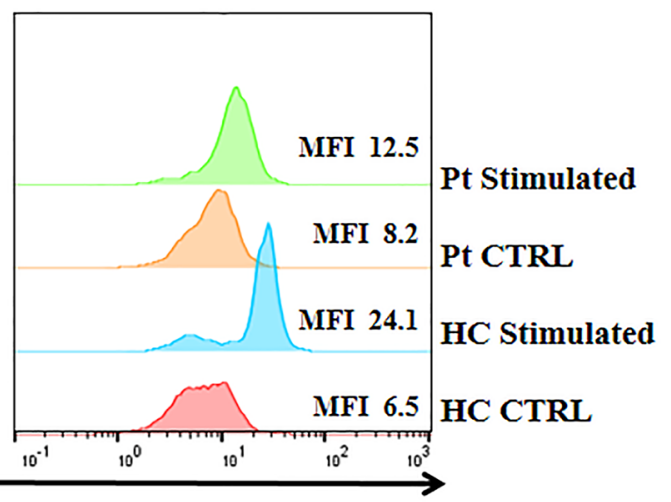

CD11b MFI
Fig. 2 Neutrophils purified from peripheral blood of asthma patients (red bars) and healthy controls (white bars) were stimulated with LPS $(100 \mathrm{ng} / \mathrm{mL})(\mathbf{A})$, fMLP $(1 \mu \mathrm{M})(\mathbf{B})$ or PMA $(10 \mathrm{ng} / \mathrm{mL})(\mathbf{C})$ for $60 \mathrm{~min}$ at $37^{\circ} \mathrm{C}$ and then stained for the neutrophil activation marker $\mathrm{CD} 11 \mathrm{~b}$ and subjected to cytofluorimetric analysis. Mean fluorescence intensity (MFI) was calculated and normalized for non-stimulated cells (control). The control was set as 1 (dashed line). Results were expressed as fold increase versus control (mean \pm SEM); $* p<0.05$;
$* * * p<0.005$. Student's $\mathrm{t}$ test or Mann-Whitney $\mathrm{U}$ test according to the parametric or nonparametric distribution of the variables. D-F. Representative histograms illustrating MFI for CD11b expression on peripheral blood neutrophils from healthy controls (HC) and asthma patients (Pt), unstimulated (CTRL) or stimulated with LPS (D), fMLP (E) or PMA (F). MFI=Mean fluorescence intensity. $\mathrm{CTRL}=$ Control cells; $\mathrm{HC}=$ Healthy controls. $\mathrm{Pt}=$ Patients 

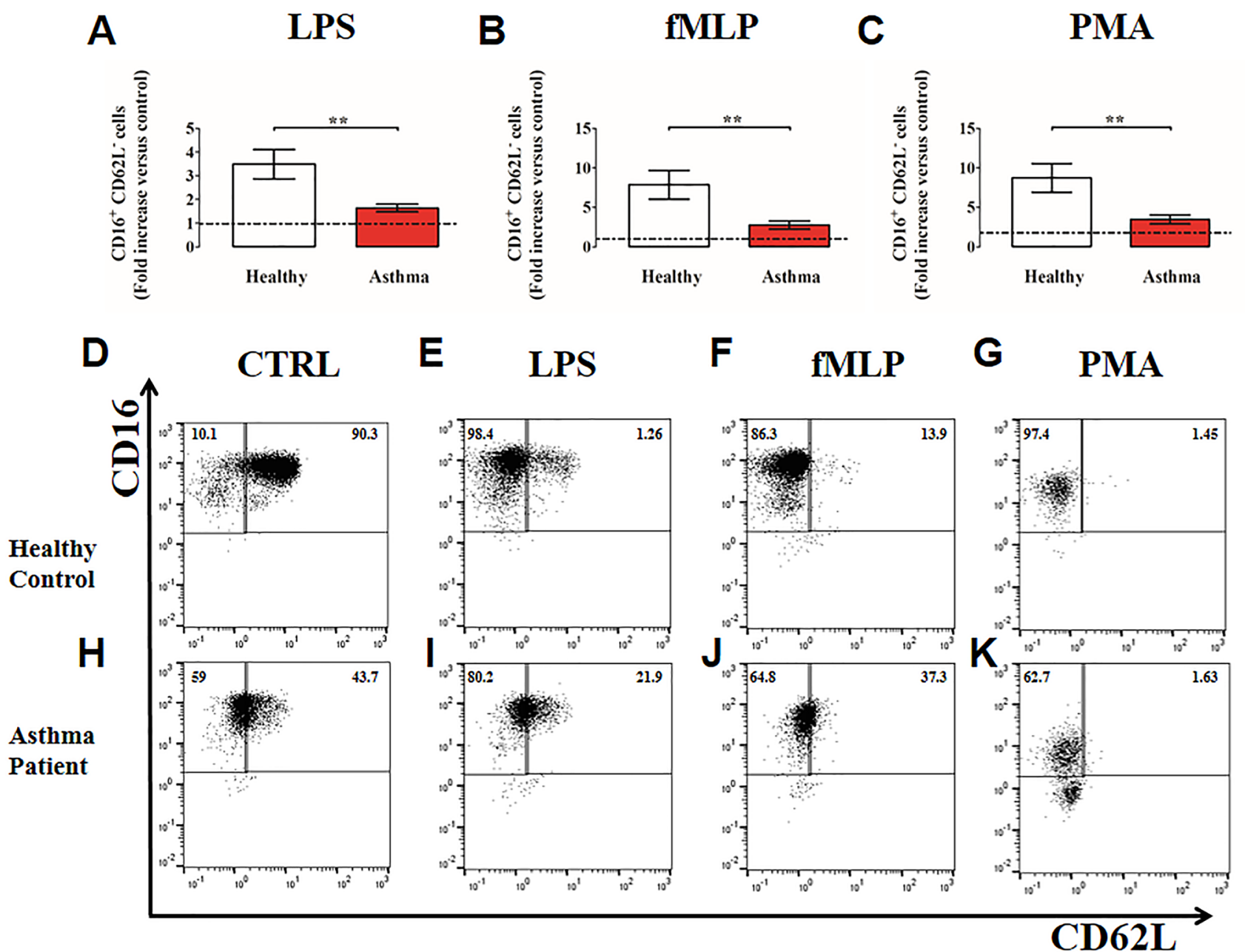

Fig. 3 Neutrophils purified from peripheral blood of asthma patients (red bars) and healthy controls (white bars) were stimulated with LPS $(100 \mathrm{ng} / \mathrm{mL})(\mathbf{A})$, fMLP $(1 \mu \mathrm{M})(\mathbf{B})$ or PMA $(10 \mathrm{ng} / \mathrm{mL})(\mathbf{C})$ or control medium for $60 \mathrm{~min}$ at $37^{\circ} \mathrm{C}$ and then stained for neutrophil activation markers $\mathrm{CD} 16$ and $\mathrm{CD} 62 \mathrm{~L}$ and subjected to cytofluorimetric analysis. Percentages of $\mathrm{CD}_{16}{ }^{+} \mathrm{CD}_{2} \mathrm{~L}^{-}$cells were calculated and normalized for controls. The control was set as 1 (dashed line). The

VEGF-A, the most important angiogenic factor [85-87], is produced by activated human neutrophils $[66,88,89]$. Figure 4 shows that the plasma levels of MPO, MMP-9 and CXCL8 were significantly increased in asthmatics compared to healthy donors. By contrast, the plasma concentrations of GM-CSF and VEGF-A did not differ between the two groups of subjects examined. Collectively, these results suggest that neutrophils from asthmatics are activated in vivo to release neutrophil-derived mediators.

\section{Plasma levels of NET components}

Human neutrophils activated by a variety of immunologic and non-immunologic stimuli release NETs [74, 75, 90, 91]. We evaluated the plasma levels of different components of results were expressed as fold increase versus control (mean \pm SEM); ** $p<0.01$. Student's t test or Mann-Whitney U test according to the parametric or nonparametric distribution of the variables. D-K: Representative flow cytometric panels with respect to the gating strategy and cell counts for healthy controls $(\mathbf{D}, \mathbf{E}, \mathbf{F}, \mathbf{G})$ and asthma patients $(\mathbf{H}, \mathbf{I}, \mathbf{J}, \mathbf{K})$, stimulated with control medium $(\mathbf{D}, \mathbf{H})$, LPS $(\mathbf{E}, \mathbf{I})$, fMLP $(\mathbf{F}, \mathbf{J})$ or PMA $(\mathbf{G}, \mathbf{K})$

NETs such as circulating free DNA [42] [92] and citrullinated histone H3 (CitH3), a more specific marker for NET formation [93]. Figure 5 shows that circulating levels of dsDNA were higher in asthma patients compared to healthy donors (Fig. 5A). Even more marked were the increased plasma levels of $\mathrm{CitH} 3$ in asthmatics compared to controls (Fig. 5B). Interestingly, the circulating concentrations of $\mathrm{CitH} 3$ were inversely correlated with a parameter $\mathrm{FEV}_{1} / \mathrm{FVC}$ of lung function in asthmatics (Fig. 6). 
A

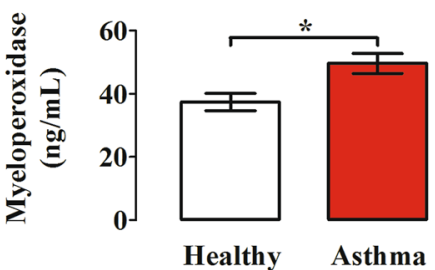

D

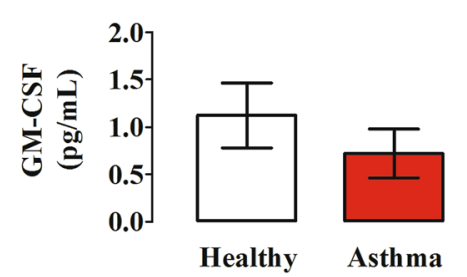

Fig. 4 Plasma concentrations of myeloperoxidase (A), MMP-9 (B), CXCL8 (C), GM-CSF (D) and VEGF-A (E) in asthma patients (red bars) and healthy controls (white bars) were measured by ELISA. The
A

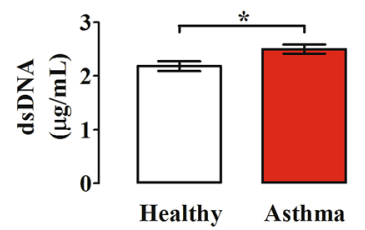

B

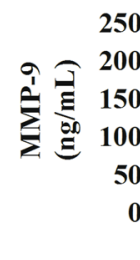

E

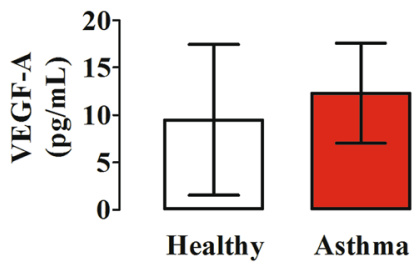

C
Fig. 5 Plasma concentrations of circulating free DNA (cfDNA) (A) and citrullinated histone $\mathrm{H} 3$ (CitH3) (B) in asthma patients (red bars) and healthy controls (white bars) were measured by Quant-iT ${ }^{\mathrm{TM}}$ PicoGreen $^{\mathrm{TM}}$ dsDNA Assay Kit (Thermo Fisher) and Citrullinated Histone H3 (clone 11D3) ELISA kit (Cayman), respectively. Results were expressed as mean $\pm \mathrm{SEM} ; * p<0.05 ; * * * p<0.005$. Student's $\mathrm{t}$ test or Mann-Whitney U test according to the parametric or nonparametric distribution of the variables

\section{Discussion}

Highly purified neutrophils isolated from peripheral blood of asthma patients and healthy donors produced ROS in response to two agonists (LPS and fMLP) that activate specific receptors (TLR4 and FPR, respectively) expressed on the cytoplasmic membrane of these cells $[24,66]$. The production of ROS was reduced in PMN from asthmatics compared to healthy donors. Similarly, the mobilization of two surface markers of neutrophils, CD11b and CD62L, induced by LPS and fMLP, were more marked in cells from healthy donors compared to asthmatics. Collectively, these results are compatible with the hypothesis that circulating neutrophils from asthma patients are

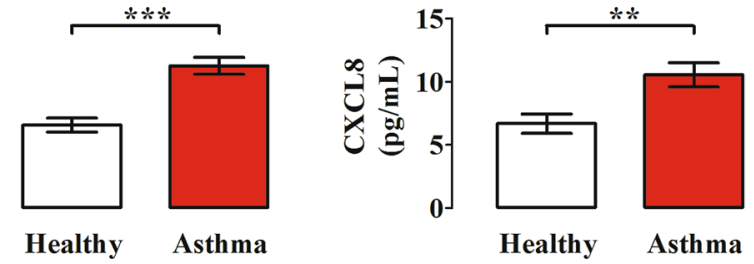

results were expressed as mean \pm SEM. $* p<0.05$; ** $p<0.01$; *** $p<0.005$. Student's t test or Mann-Whitney U test according to the parametric or nonparametric distribution of the variables

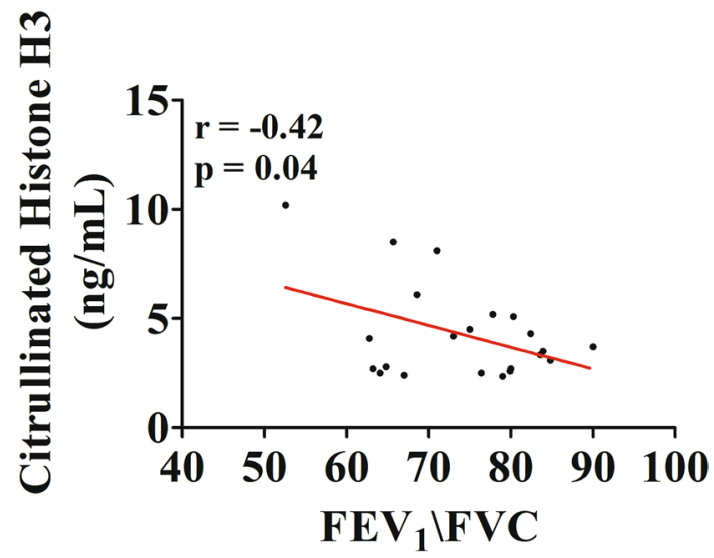

Fig. 6 Correlation between plasma concentrations of Citrullinated Histone $\mathrm{H} 3$ (CitH3 and $\mathrm{FEV}_{1} / \mathrm{FVC}(\%)$ in asthma patients. Spearman correlation coefficient with $r=-0.42 ;{ }^{*} p<0.05$

in vivo activated and desensitized. Circulating levels of neutrophil-derived mediators, MPO, MMP-9 and CXCL8, were increased in asthmatics compared to controls. Plasma levels of two NET components, dsDNA and CitH3, a specific measure for NET formation, were increased in asthmatics and inversely correlated with a parameter of lung function in asthmatics.

Human neutrophils are a prominent source of ROS, being capable of producing significant quantities of superoxide anion $\left(\mathrm{O}_{2}^{-}\right)$, hydrogen peroxide $\left(\mathrm{H}_{2} \mathrm{O}_{2}\right)$ and hypochlorous acid (HOCl) [23, 94]. These species are indispensable component of host defense against microorganisms, but are also capable of tissue damage in different murine models of asthma $[52,95]$. A limited study performed in 8 asthmatics 
and 7 controls reported that fMLP- and PMA-induced $\mathrm{O}_{2}{ }^{-}$production by circulating neutrophils was increased in asthma patients [96]. A similar study in 11 asthmatics confirmed that fMLP- and PMA-induced production of $\mathrm{O}_{2}{ }^{-}$by neutrophils was greater in asthma patients [97]. In asthmatics, the production of $\mathrm{O}_{2}^{-}$inversely correlated with $\mathrm{FEV}_{1}$. More recently, it has been shown that peripheral blood neutrophils from asthmatics activated by fMLP and LPS induced an increase in ROS production [49]. Our results confirm and extend the previous observations by showing that two receptor-mediated stimuli (i.e., fMLP and LPS) and PMA, a direct activator of neutrophil PKC, caused a time-dependent production of ROS from highly purified neutrophil isolated from asthmatics and normal subjects. However, differently from previous studies [96, 97], the production of ROS in PMNs from asthmatics induced by the three stimuli was decreased compared to normal donors. Different experimental techniques and study design might explain, at least in part, these apparently different results. For instance, previous studies evaluated the $\mathrm{O}_{2}{ }^{-}$generation by human neutrophils [96, 97], whereas we and others [49] have measured the intracellular production of ROS in highly purified neutrophils obtained from larger cohorts of donors. Alternatively, there is the possibility that the pharmacologic treatment of asthma patients may have altered the production of ROS from neutrophils. For instance, ICS and/or oral glucocorticoid treatment in the vast majority $(>80 \%)$ of our patients may have reduced ROS production from neutrophils. We would like to suggest that circulating neutrophils from asthmatics are in vivo chronically stimulated by various stimuli which render these cells hyporesponsive to in vitro stimulation.

To support this hypothesis, we evaluated the changes of the neutrophil expression of CD11b and CD62L induced by LPS, fMLP and PMA in asthmatics and controls. Under basal conditions, there is a minimal surface expression of CD11b [60]. All three stimuli activated neutrophils inducing the over-expression of CD11b, which was more marked in healthy controls compared to asthma patients. CD62L is normally expressed by the majority of resting neutrophils and is down-regulated following cell activation. The three stimuli induced a more marked CD62L shedding in neutrophils from healthy donors compared to asthmatics. Collectively, these results support the hypothesis that neutrophils from asthmatics are presumably in vivo desensitized and are less responsive to in vitro activation by various stimuli.

Our experiments were performed on normal density neutrophils (NDNs) isolated by Ficoll-Paque Histopaque ${ }^{\circledR}$ density gradient centrifugation. Several groups of investigators have identified and characterized a subset of human peripheral blood PMNs named low-density neutrophils (LDNs) [70, 98-101]. It has been suggested that LDNs represent a subset of activated neutrophils compared to NDNs [102].
Interestingly, fMLP selectively up-regulated CD11b and down-regulated CD62L expression in LDNs. Based on these findings, we cannot exclude the possibility that some of the differences that we found in the LPS/fMLP/PMA-induced expression of $\mathrm{CD} 11 \mathrm{~b}$ and/or CD62L in neutrophils could be due to changes in the percentage of NDNs and LDNs in asthmatics.

Neutrophils are a rich source of preformed granular constituents which can be rapidly released [23]. MPO is a cationic enzyme localized in primary azurophilic granules, which catalyzes ROS production [73] and contributes to NET formation $[24,103]$. In this study, we found that plasma levels of MPO are increased in asthmatics compared to healthy controls. A recent study demonstrated that sputum concentrations of MPO are increased in patients with asthma and correlated with a NET-derived biomarker [45].

MMP-9, present in tertiary granules of neutrophils, was found in BAL of asthmatics [79] and it was correlated to the absolute neutrophil count [104], MMP- $9^{-/-}$mice showed reduced immune cell infiltration and bronchial hyperresponsiveness compared to wild type mice in a murine model of asthma [76]. MMP-9 was increased in BAL and in sputum of asthmatics after allergen challenge [77, 78]. Moreover, neutrophil-derived MMP-9 was increased in severe asthma [104]. In this study, we found that plasma concentrations of MMP-9 are increased in asthma patients compared to controls.

Human neutrophils contain and release CXCL8 [105]. Asthmatic individuals have higher circulating [81, 106-108], sputum [109] and BAL fluid [82] CXCL8 concentrations compared to healthy controls. Our results indicate that plasma levels of CXCL8 are increased in asthmatics compared to healthy controls. Collectively, these results showing that three different neutrophil-derived mediators are increased in asthma patients suggest that PMNs are in vivo activated.

GM-CSF is a pleiotropic cytokine that plays a role in the differentiation, activation and survival of several immune cells, including neutrophils [110]. GM-CSF levels are higher in sputum, BAL fluid and bronchial tissue in patients with asthma [111-113]. Recent studies indicate that GM-CSF plays a central role in experimental models of asthma [83, 84]. In our cohort of asthmatics, circulating levels of GM$\mathrm{CSF}$ are comparable to controls. It is conceivable that larger patient cohorts may reveal significant differences. Alternatively, it is possible to hypothesize that GM-CSF plays a role in asthma as a local (e.g., pulmonary) rather than a circulating cytokine.

Angiogenesis, the formation of new blood vessels, is a complex process modulated by a plethora of stimulatory and inhibitory factors $[85,86]$. There is some evidence the angiogenic switch in asthmatic airways involves the production of direct (e.g., VEGFs, angiopoietins) and indirect angiogenic 
factors (e.g., MMP-9, cysteinyl leukotrienes) [86, 114, 115]. Interestingly, the plasma concentrations of VEGF-A, which is also released by activated human PMNs [88, 89], were similar in asthmatic and controls. Our finding that circulating levels of VEGF-A are not altered in asthma patients compared to healthy donors is rather intriguing. In fact, several immune cells involved in the pathogenesis of asthma such as mast cells [116, 117], macrophages [118], basophils [119], eosinophils [120] and neutrophils [66, 88, 89] are a major source of VEGF-A. Perhaps, the local production of angiogenic factors is more important than circulating levels of these factors in asthma. An alternative hypothesis to explain our results could arise from the observation that ROS up-regulate VEGF-A expression [121]. Therefore, the reduced production of ROS from neutrophils of asthmatics could explain, at least in part, the normal plasma levels of VEGF-A in these patients.

NETs were originally described as a protective mechanism by which neutrophils exerted an antibacterial role $[25,74]$. During the last years, this initial interpretation has significantly evolved by showing that NETs can exert proinflammatory [24], as well as anti-inflammatory effects [122]. Moreover, human neutrophils can release lytic [25, 123] and non-lytic NETs [60, 91, 124]. There is growing evidence that NETs play multiple roles in several inflammatory diseases [37, 38, 125], cancer [24, 60, 92] and allergic disorders [42, 43, 45, 47, 126, 127]. NETs were first described in bronchial biopsy specimens from four atopic asthmatics expressing a high number of neutrophils [47]. dsDNA, a putative marker of NET formation, was found in induced sputum of asthmatics [126]. Johnston and collaborators demonstrated that nasal dsDNA levels were increased in nasal lavage after rhinovirus infection in asthmatics [42]. A collaborative study on severe asthma found that BAL dsDNA was increased in patients with neutrophilia [127]. A similar study reported that increased dsDNA in sputum from patients with severe asthma reflected local neutrophil activation [45]. Interestingly, a positive linear correlation was found between dsDNA and MPO concentrations. The latter observation is relevant because MPO is a key component of NET formation [29].

Different analytical methods (e.g., dsDNA, MPO-DNA, $\mathrm{CitH} 3$ ) for the measurement of NET markers in asthma were used by several groups $[42,45,126,128-130]$. The measurement of dsDNA has been widely used as a NET marker [42, $45,126]$. However, the quantitative analysis of circulating DNA does not necessarily reflect the extent of NET formation in vivo. In fact, DNA complexes may result from any cell death associated with neutrophilic inflammation [128]. MPO-DNA has been proposed as a circulating NET marker of severe asthma [130]. However, the ELISA detection of MPO-DNA complexes in human plasma is error prone and yields limited information of NETs formed in vivo [129].
For the above reasons, we have used two different analytical methods (i.e., dsDNA and $\mathrm{CitH} 3$ ). Our results demonstrate that plasma concentrations of two putative markers of NETs, dsDNA and CitH3, are increased in asthma patients compared to healthy controls. In particular, we validated our findings by measuring plasma levels of $\mathrm{CitH} 3$ which is considered a more reliable biomarker of NET formation [24]. Collectively, these results indicate that increased circulating levels of two markers of NET formation are associated with bronchial asthma.

Putative markers of NETs have been detected in different sites/biological fluids of asthmatics. DNA presence was initially described in bronchial biopsies of adult asthmatics [47]. dsDNA have been found in sputum [45, 126], nasal lavages [42] and BAL of asthma patients [127, 130]. Granger et al. recently investigated the presence of putative NET markers in serum of asthma patients [130]. These authors examined MPO-DNA complexes by ELISA which is error prone and yields limited information of NETs in vivo [129]. Our study is, to the best of our knowledge, the only one in which two different analytical methods (i.e., dsDNA and $\mathrm{CitH} 3$ ) are used to evaluate plasma NETs in asthmatic patients. It is evident that the ease of access to plasma samples compared to BAL or induced sputum makes our results of potential clinical applicability.

Our findings might have additional translational relevance for a number of possible reasons. First, there is the possibility that measurement of circulation NET-derived products (i.e., CitH3) could represent an easily measurable plasma biomarker of asthma severity. In this context, we have found an inverse correlation between circulating levels of $\mathrm{CitH} 3$ and $\mathrm{FEV}_{1} / \mathrm{FVC}$ in asthmatics. Second, several inhibitors of NET formation and molecules that degrade NETs are under active investigation for the treatment of inflammatory disorders and cancer [24]. If further studies will demonstrate the pathogenic role of NETs in asthma, it is conceivable that some of the various strategies that modulate NET formation [24] could be envisioned for the treatment of allergic disorders.

At this point, the question obviously arises: what could be the stimuli that induce the in vivo formation of NETs in asthmatics? A plethora of immunologic and non-immunologic stimuli can induce NET formation in vivo. Among these, LPS [60, 75, 131, 132], IL-17 [133], IL-33 [134, 135], CXCL8 [60, 136-138], C3a/C5a [75, 139, 140] and IL-1 $\beta$ $[45,141]$, which have been implicated in various aspects of asthma [42, 45], are inducers of NET formation in neutrophils. Moreover, several bacterial products (i.e., fMLP) [92, $139,142]$, rhinovirus and influenza virus can induce NET formation $[42,143]$. Further studies are necessary to identify in vivo inducers of NET formation in asthmatics.

T2-high asthma is characterized by type 2 inflammation involving Th2 lymphocytes, ILC2s, mast cells, basophils, 
eosinophils, type 2 cytokines and IgE [8-10]. On the other hand, T2-low asthma is less characterized and might involve Th1 and/or Th17 cells, ILC1s and ILC3s $[9,15]$. There is increasing evidence that this is a rather simplistic and procrustean classification. Our results emphasize a possible role of NETs and neutrophil-derived mediators also in allergic asthmatics. These findings could suggest that neutrophils could play a pathogenic role not only in T2-low but also in a percentage of T2-high asthmatics.

This study has several limitations that should be pointed out. The sample size of the asthma patient and healthy control cohorts investigated in the present study is limited. Further studies on larger cohorts of healthy donors and patients could highlight the significance of NET formation in these patients. Although our results indicate that increased CitH3 plasma levels were inversely correlated to reduced lung function, at this stage, it would have been inappropriate to define $\mathrm{CitH} 3$ as a biomarker of asthma severity. We anticipate that it would be necessary to examine at least one order of magnitude larger cohorts. Asthma is a heterogeneous group of inflammatory disorders characterized by complex pathology, distinct subtypes and highly variable clinical courses [144-147]. The patients included in this study were adults with a history of allergist-diagnosed asthma of different severity (mild, moderate, severe). No correlation was found between the plasma CitH3 concentrations and FeNO, serum IgE and peripheral blood eosinophils or neutrophils. The limited sample size of patients does not allow to correlate the increased CitH3 levels to T2-low or T2-high asthma. The contribution of NETs and the clinical relevance of CitH3 measurement as a biomarker in different forms of asthma remain to be investigated in larger cohorts of patients. Finally, two forms of NET formation have been identified: suicidal NETosis [25, 123] and vital NET release [60, 91, 124]. Further in vitro and in vivo studies should investigate which of these two distinct forms of NET formation are involved in different phenotypes of asthma.

In conclusion, our study indicates that circulating neutrophils from asthmatics released ROS when activated by immunologic and non-immunologic stimuli. Plasma levels of several biomarkers of neutrophils activation such as MPO, MMP-9 and CXCL8 were increased in asthma patients compared to healthy subjects. Circulating levels of two NET-derived products such as dsDNA and $\mathrm{CitH} 3$ were increased in asthmatics compared to controls and were inversely correlated to $\mathrm{FEV}_{1} / \mathrm{FVC}$ decrease. Together, these findings indicate that neutrophils and their products might have an active role in the pathophysiology of asthma.

Supplementary Information The online version contains supplementary material available at https://doi.org/10.1007/s10238-021-00750-8.
Acknowledgements We would like to thank Dr. Gjada Criscuolo for critical reading of the manuscript and the administrative staff (Dr. Roberto Bifulco, Dr. Anna Ferraro and Dr. Maria Cristina Fucci), without whom it would not be possible to work as a team.

Funding Open access funding provided by Università degli Studi di Napoli Federico II within the CRUI-CARE Agreement. This work was supported in part by grants from the CISI-Lab Project (University of Naples Federico II), TIMING Project and Campania Bioscience (Regione Campania) and by MIUR PRIN 2017 M8Y MR8_005. (M.R.G.).

Data Availability All data generated or analyzed during this study are included in this published article.

\section{Declarations}

Conflicts of interest The authors declare that they do not have any conflict of interest.

Ethical approval The study has received ethical, regulatory and institutional approvals from Ethics Committee of the University of Naples Federico II (Protocol Number: 198/18) and was run in accordance with the recommendation from the Declaration of Helsinki. All participants or their legal representatives provided informed consent before their enrollment in the registry.

Consent to participate Informed consent was obtained from all subjects involved in the study.

Open Access This article is licensed under a Creative Commons Attribution 4.0 International License, which permits use, sharing, adaptation, distribution and reproduction in any medium or format, as long as you give appropriate credit to the original author(s) and the source, provide a link to the Creative Commons licence, and indicate if changes were made. The images or other third party material in this article are included in the article's Creative Commons licence, unless indicated otherwise in a credit line to the material. If material is not included in the article's Creative Commons licence and your intended use is not permitted by statutory regulation or exceeds the permitted use, you will need to obtain permission directly from the copyright holder. To view a copy of this licence, visit http://creativecommons.org/licenses/by/4.0/.

\section{References}

1. Kaur R, Chupp G. Phenotypes and endotypes of adult asthma: Moving toward precision medicine. J Allergy Clin Immunol. 2019;144(1):1-12.

2. Asthma WHO, 2019. https://www.who.int/news-room/q-adetail/asthma.

3. Chung KF, Wenzel SE, Brozek JL, et al. International ERS/ ATS guidelines on definition, evaluation and treatment of severe asthma. Eur Respir J. 2014;43(2):343-73.

4. Canonica GW, Senna G, Mitchell PD, et al. Therapeutic interventions in severe asthma. World Allergy Organ J. 2016;9(1):40.

5. Lotvall J, Akdis CA, Bacharier LB, et al. Asthma endotypes: a new approach to classification of disease entities within the asthma syndrome. J Allergy Clin Immunol. 2011;127(2):355-60. 
6. Ferrando M, Bagnasco D, Varricchi G, et al. Personalized Medicine in Allergy. Allergy Asthma Immunol Res. 2017;9(1):15-24.

7. Tabatabaian F, Ledford DK, Casale TB. Biologic and new therapies in asthma. Immunol Allergy Clin North Am. 2017;37(2):329-43.

8. Marone G, Spadaro G, Braile M, et al. Tezepelumab: a novel biological therapy for the treatment of severe uncontrolled asthma. Expert Opin Investig Drugs. 2019;28(11):931-40.

9. Pepper AN, Renz H, Casale TB, Garn H. Biologic therapy and novel molecular targets of severe asthma. J Allergy Clin Immunol Pract. 2017;5(4):909-16.

10. Marone G, Borriello F, Varricchi G, Genovese A, Granata F. Basophils: historical reflections and perspectives. Chem Immunol Allergy. 2014;100:172-92.

11. Varricchi G, Harker J, Borriello F, et al. T follicular helper (Tfh ) cells in normal immune responses and in allergic disorders. Allergy. 2016;71(8):1086-94.

12. Gong F, Zheng T, Zhou P. T follicular helper cell subsets and the associated cytokine il-21 in the pathogenesis and therapy of asthma. Front Immunol. 2019;10:2918.

13. Kuruvilla ME, Lee FE, Lee GB. Understanding asthma phenotypes, endotypes, and mechanisms of disease. Clin Rev Allergy Immunol. 2019;56(2):219-33.

14. Bel EH, Ten Brinke A. New anti-eosinophil drugs for asthma and COPD: targeting the trait! Chest. 2017;152(6):1276-82.

15. Ricciardolo FLM, Sorbello V, Folino A, et al. Identification of IL-17F/frequent exacerbator endotype in asthma. J Allergy Clin Immunol. 2017;140(2):395-406.

16. Samitas K, Zervas E, Gaga M. T2-low asthma: current approach to diagnosis and therapy. Curr Opin Pulm Med. 2017;23(1):48-55.

17. Ray A, Kolls JK. Neutrophilic inflammation in asthma and association with disease severity. Trends Immunol. 2017;38(12):942-54.

18. Fitzpatrick AM, Chipps BE, Holguin F, Woodruff PG. T2-"Low" Asthma: overview and management strategies. J Allergy Clin Immunol Pract. 2020;8(2):452-63.

19. Lee Y, Quoc QL, Park HS. Biomarkers for severe asthma: lessons from longitudinal cohort studies. Allergy Asthma Immunol Res. 2021;13(3):375-89.

20. Camicia G, Pozner R, de Larranaga G. Neutrophil extracellular traps in sepsis. Shock. 2014;42(4):286-94.

21. Galdiero MR, Varricchi G, Seaf M, Marone G, Levi-Schaffer F. Bidirectional mast cell-eosinophil interactions in inflammatory disorders and cancer. Front Med (Lausanne). 2017;4:103.

22. Kolaczkowska E, Kubes P. Neutrophil recruitment and function in health and inflammation. Nat Rev Immunol. 2013;13(3):159-75.

23. Lehman HK, Segal BH. The role of neutrophils in host defense and disease. J Allergy Clin Immunol. 2020;145(6):1535-44.

24. Cristinziano L, Modestino L, Antonelli A, et al. Neutrophil Extracellular Traps in Cancer. Sem Cancer Biol. 2021. https:// doi.org/10.1016/j.semcancer.2021.07.011.

25. Brinkmann V, Reichard U, Goosmann C, et al. Neutrophil extracellular traps kill bacteria. Science. 2004;303(5663):1532-5.

26. Pittman K, Kubes P. Damage-associated molecular patterns control neutrophil recruitment. J Innate Immun. 2013;5(4):315-23.

27. Weiss E, Kretschmer D. Formyl-Peptide Receptors in Infection, Inflammation, and Cancer. Trends Immunol. 2018;39(10):815-29.

28. Mantovani A, Cassatella MA, Costantini C, Jaillon S. Neutrophils in the activation and regulation of innate and adaptive immunity. Nat Rev Immunol. 2011;11(8):519-31.

29. Arnhold J. The dual role of myeloperoxidase in immune response. Int J Mol Sci. 2020;21(21):8057.
30. Urban CF, Reichard U, Brinkmann V, Zychlinsky A. Neutrophil extracellular traps capture and kill Candida albicans yeast and hyphal forms. Cell Microbiol. 2006;8(4):668-76.

31. Saitoh T, Komano J, Saitoh Y, et al. Neutrophil extracellular traps mediate a host defense response to human immunodeficiency virus-1. Cell Host Microbe. 2012;12(1):109-16.

32. Zuo, Y., Yalavarthi, S., Shi, H., et al., Neutrophil extracellular traps in COVID-19. JCI Insight, 2020; 5(11):e138999.

33. Raftery MJ, Lalwani P, Krautkrmer E, et al. beta2 integrin mediates hantavirus-induced release of neutrophil extracellular traps. J Exp Med. 2014;211(7):1485-97.

34. Yousefi S, Gold JA, Andina N, et al. Catapult-like release of mitochondrial DNA by eosinophils contributes to antibacterial defense. Nat Med. 2008;14(9):949-53.

35. Amini P, Stojkov D, Felser A, et al. Neutrophil extracellular trap formation requires OPA1-dependent glycolytic ATP production. Nat Commun. 2018;9(1):2958.

36. Skendros P, Mitsios A, Chrysanthopoulou A, et al. Complement and tissue factor-enriched neutrophil extracellular traps are key drivers in COVID-19 immunothrombosis. J Clin Invest. 2020;130(11):6151-7.

37. Hakkim A, Furnrohr BG, Amann K, et al. Impairment of neutrophil extracellular trap degradation is associated with lupus nephritis. Proc Natl Acad Sci U S A. 2010;107(21):9813-8.

38. van den Hoogen LL, van der Linden M, Meyaard L, et al. Neutrophil extracellular traps and low-density granulocytes are associated with the interferon signature in systemic lupus erythematosus, but not in antiphospholipid syndrome. Ann Rheum Dis. 2020;79(10):e135.

39. Berger-Achituv S, Brinkmann V, Abed UA, et al. A proposed role for neutrophil extracellular traps in cancer immunoediting. Front Immunol. 2013;4:48.

40. Gregory AD, Houghton AM. Tumor-associated neutrophils: new targets for cancer therapy. Cancer Res. 2011;71(7):2411-6.

41. Cools-Lartigue J, Spicer J, Najmeh S, Ferri L. Neutrophil extracellular traps in cancer progression. Cell Mol Life Sci. 2014;71(21):4179-94.

42. Toussaint M, Jackson DJ, Swieboda D, et al. Host DNA released by NETosis promotes rhinovirus-induced type- 2 allergic asthma exacerbation. Nat Med. 2017;23(6):681-91.

43. Hudock KM, Collins MS, Imbrogno M, et al. Neutrophil extracellular traps activate IL-8 and IL-1 expression in human bronchial epithelia. Am J Physiol Lung Cell Mol Physiol. 2020;319(1):L137-47.

44. Chen X, Li Y, Qin L, He R, Hu C. Neutrophil extracellular trapping network promotes the pathogenesis of neutrophil-associated asthma through macrophages. Immunol Invest. 2020;50(5):1-18.

45. Lachowicz-Scroggins ME, Dunican EM, Charbit AR, et al. Extracellular DNA, neutrophil extracellular traps, and inflammasome activation in severe asthma. Am J Respir Crit Care Med. 2019;199(9): 1076-85.

46. Wan R, Jiang J, Hu C, et al. Neutrophil extracellular traps amplify neutrophil recruitment and inflammation in neutrophilic asthma by stimulating the airway epithelial cells to activate the TLR4/ NF-kappaB pathway and secrete chemokines. Aging (Albany NY). 2020;12(17):16820-36.

47. Dworski R, Simon HU, Hoskins A, Yousefi S. Eosinophil and neutrophil extracellular DNA traps in human allergic asthmatic airways. J Allergy Clin Immunol. 2011;127(5):1260-6.

48. Pelaia G, Vatrella A, Busceti MT, et al. Cellular mechanisms underlying eosinophilic and neutrophilic airway inflammation in asthma. Mediators Inflamm. 2015;2015:879783.

49. Kim SH, Uuganbayar U, Trinh HKT, et al. Evaluation of Neutrophil Activation Status According to the Phenotypes of Adult Asthma. Allergy Asthma Immunol Res. 2019;11(3):381-93. 
50. Ekstedt S, Stenberg H, Tufvesson E, et al. The potential role of CD16(high) CD62L(dim) neutrophils in the allergic asthma. Allergy. 2019;74(11):2265-8.

51. Qi L, Xiangdong Z, Hongmei Y, Xiaohong N, Xiaoyan X. Roles of ROS/TACE in neutrophil elastase-induced mucus hypersecretion in NCI-H292 airway epithelial cells. Eur Cytokine Netw. 2010;21(3):177-85.

52. Liu YN, Zha WJ, Ma Y, et al. Galangin attenuates airway remodelling by inhibiting TGF-beta1-mediated ROS generation and MAPK/Akt phosphorylation in asthma. Sci Rep. 2015;5:11758.

53. Karacs J, Reithofer M, Kitzmuller C, et al. Adjuvants and vaccines used in allergen-specific immunotherapy induce neutrophil extracellular traps. Vaccines. 2021;9(4):321.

54. Detoraki A, Di Capua L, Varricchi G, et al. Omalizumab in patients with eosinophilic granulomatosis with polyangiitis: a 36-month follow-up study. J Asthma. 2016;53(2):201-6.

55. Bagnasco D, Ferrando M, Varricchi G, et al. Anti-Interleukin 5 (IL-5) and IL-5Ra Biological Drugs: Efficacy, Safety, and Future Perspectives in Severe Eosinophilic Asthma. Front Med (Lausanne). 2017;4:135

56. Schatz M, Sorkness CA, Li JT, et al. Asthma Control Test: reliability, validity, and responsiveness in patients not previously followed by asthma specialists. J Allergy Clin Immunol. 2006;117(3):549-56.

57. Graham BL, Steenbruggen I, Miller MR, et al. Standardization of spirometry 2019 update. An official american thoracic society and european respiratory society technical statement. Am J Respir Crit Care Med. 2019;200(8):e70-88.

58. Quanjer PH, Stanojevic S, Cole TJ, et al. Multi-ethnic reference values for spirometry for the 3-95-yr age range: the global lung function 2012 equations. Eur Respir J. 2012;40(6):1324-43.

59. American Thoracic Society/European Respiratory Society recommendations for standardized procedures for the online and offline measurement of exhaled lower respiratory nitric oxide and nasal nitric oxide, 2005. Am J Respir Crit Care Med, 2005; 171(8): 912-30.

60. Cristinziano L, Modestino L, Loffredo S, et al. Anaplastic thyroid cancer cells induce the release of mitochondrial extracellular DNA traps by viable neutrophils. J Immunol. 2020;204(5):1362-72.

61. Calzetti F, Tamassia N, Arruda-Silva F, Gasperini S, Cassatella MA. The importance of being "pure" neutrophils. J Allergy Clin Immunol. 2017;139(1):352-5.

62. Borriello F, Iannone R, Di Somma S, et al. GM-CSF and IL-3 modulate human monocyte tnf-alpha production and renewal in in vitro models of trained immunity. Front Immunol. 2016;7:680.

63. Demers M, Wagner DD. NETosis: a new factor in tumor progression and cancer-associated thrombosis. Semin Thromb Hemost. 2014;40(3):277-83.

64. van Montfoort ML, Stephan F, Lauw MN, et al. Circulating nucleosomes and neutrophil activation as risk factors for deep vein thrombosis. Arterioscler Thromb Vasc Biol. 2013;33(1):147-51.

65. Goud PT, Bai D, Abu-Soud HM. A Multiple-hit hypothesis involving reactive oxygen species and myeloperoxidase explains clinical deterioration and fatality in COVID-19. Int J Biol Sci. 2021;17(1):62-72.

66. Braile M, Cristinziano L, Marcella S, et al. LPS-mediated neutrophil VEGF-A release is modulated by cannabinoid receptor activation. J Leukoc Biol. 2021;109(3):621-31.

67. Stocks SC, Ruchaud-Sparagano MH, Kerr MA, et al. CD66: role in the regulation of neutrophil effector function. Eur J Immunol. 1996;26(12):2924-32.

68. Condliffe AM, Chilvers ER, Haslett C, Dransfield I. Priming differentially regulates neutrophil adhesion molecule expression/ function. Immunology. 1996;89(1):105-11.
69. Galdiero MR, Varricchi G, Loffredo S, et al. Potential involvement of neutrophils in human thyroid cancer. PLoS One. 2018;13(6):e0199740.

70. Pillay J, Kamp VM, van Hoffen E, et al. A subset of neutrophils in human systemic inflammation inhibits $\mathrm{T}$ cell responses through Mac-1. J Clin Invest. 2012;122(1):327-36.

71. Cassatella MA, Ostberg NK, Tamassia N, Soehnlein O. Biological Roles of Neutrophil-Derived Granule Proteins and Cytokines. Trends Immunol. 2019;40(7):648-64.

72. Hendrix AY, Kheradmand F. The Role of Matrix Metalloproteinases in Development, Repair, and Destruction of the Lungs. Prog Mol Biol Transl Sci. 2017;148:1-29.

73. Aratani Y. Myeloperoxidase: Its role for host defense, inflammation, and neutrophil function. Arch Biochem Biophys. 2018;640:47-52.

74. Brinkmann V, Zychlinsky A. Neutrophil extracellular traps: is immunity the second function of chromatin? J Cell Biol. 2012;198(5):773-83.

75. Yousefi S, Mihalache C, Kozlowski E, Schmid I, Simon HU. Viable neutrophils release mitochondrial DNA to form neutrophil extracellular traps. Cell Death Differ. 2009;16(11):1438-44.

76. Cataldo DD, Tournoy KG, Vermaelen K, et al. Matrix metalloproteinase-9 deficiency impairs cellular infiltration and bronchial hyperresponsiveness during allergen-induced airway inflammation. Am J Pathol. 2002;161(2):491-8.

77. Kelly EA, Busse WW, Jarjour NN. Increased matrix metalloproteinase-9 in the airway after allergen challenge. Am J Respir Crit Care Med. 2000;162(3 Pt 1):1157-61.

78. Cataldo DD, Bettiol J, Noel A, et al. Matrix metalloproteinase-9, but not tissue inhibitor of matrix metalloproteinase-1, increases in the sputum from allergic asthmatic patients after allergen challenge. Chest. 2002;122(5):1553-9.

79. Wenzel SE, Balzar S, Cundall M, Chu HW. Subepithelial basement membrane immunoreactivity for matrix metalloproteinase 9: association with asthma severity, neutrophilic inflammation, and wound repair. J Allergy Clin Immunol. 2003;111(6):1345-52.

80. Bajbouj K, Ramakrishnan RK, Hamid Q. Role of Matrix Metalloproteinases in Angiogenesis and Its Implications in Asthma. J Immunol Res. 2021;2021:6645072.

81. Huang AX, Lu LW, Liu WJ, Huang M. Plasma inflammatory cytokine IL-4, IL-8, IL-10, and TNF-alpha levels correlate with pulmonary function in patients with asthma-chronic obstructive pulmonary disease (COPD) overlap syndrome. Med Sci Monit. 2016;22:2800-8.

82. Hosoki K, Ying S, Corrigan C, et al. Analysis of a Panel of 48 Cytokines in BAL Fluids Specifically Identifies IL-8 Levels as the Only Cytokine that Distinguishes Controlled Asthma from Uncontrolled Asthma, and Correlates Inversely with FEV1. PLoS One. 2015;10(5):e0126035.

83. Nobs SP, Pohlmeier L, Li F, et al. GM-CSF instigates a dendritic cell-T-cell inflammatory circuit that drives chronic asthma development. J Allergy Clin Immunol. 2021;147(6):2118-33.

84. Haspeslagh E, Vanheerswynghels M, Deswarte K, et al. Prophylactic allergen immunotherapy with Der $\mathrm{p} 2$ prevents murine asthma by regulating lung GM-CSF. J Allergy Clin Immunol. 2019;143(6):2307-11.

85. Marcella, S., Petraroli, A., Braile, M., et al., Vascular endothelial growth factors and angiopoietins as new players in mastocytosis. Clin Exp Med. 2021;21(3):415-27.

86. Cristinziano L, Poto R, Criscuolo G, et al. IL-33 and superantigenic activation of human lung mast cells induce the release of angiogenic and lymphangiogenic factors. Cells. 2021;10(1):145. 
87. Sammarco G, Varricchi G, Ferraro V, et al. Mast cells, angiogenesis and lymphangiogenesis in human gastric cancer. Int J Mol Sci. 2019;20(9):2106.

88. Loffredo S, Borriello F, Iannone R, et al. Group V secreted phospholipase $\mathrm{a} 2$ induces the release of proangiogenic and antiangiogenic factors by human neutrophils. Front Immunol. 2017;8:443.

89. Loffredo S, Bova M, Suffritti C, et al. Elevated plasma levels of vascular permeability factors in $\mathrm{C} 1$ inhibitor-deficient hereditary angioedema. Allergy. 2016;71(7):989-96.

90. Pilsczek FH, Salina D, Poon KK, et al. A novel mechanism of rapid nuclear neutrophil extracellular trap formation in response to Staphylococcus aureus. J Immunol. 2010;185(12):7413-25.

91. Yousefi S, Stojkov D, Germic N, et al. Untangling "NETosis" from NETs. Eur J Immunol. 2019;49(2):221-7.

92. Albrengues J, Shields MA, Ng D, et al. Neutrophil extracellular traps produced during inflammation awaken dormant cancer cells in mice. Science. 2018;361(6409):eaao04227.

93. Yang L, Liu Q, Zhang X, et al. DNA of neutrophil extracellular traps promotes cancer metastasis via CCDC25. Nature. 2020;583(7814):133-8.

94. Snelgrove RJ, Patel DF, Patel T, Lloyd CM. The enigmatic role of the neutrophil in asthma: Friend, foe or indifferent? Clin Exp Allergy. 2018;48(10):1275-85.

95. Kim JM, Kim HK, Im YN, et al. FcgammaR/ROS/CK2alpha Is the Key Inducer of NF-kappaB Activation in a Murine Model of Asthma. Int Arch Allergy Immunol. 2018;175(1-2):16-25.

96. Meltzer S, Goldberg B, Lad P, Easton J. Superoxide generation and its modulation by adenosine in the neutrophils of subjects with asthma. J Allergy Clin Immunol. 1989;83(5):960-6.

97. Kanazawa H, Kurihara N, Hirata K, Takeda T. The role of free radicals in airway obstruction in asthmatic patients. Chest. 1991;100(5):1319-22.

98. Scapini P, Marini O, Tecchio C, Cassatella MA. Human neutrophils in the saga of cellular heterogeneity: insights and open questions. Immunol Rev. 2016;273(1):48-60.

99. Sagiv JY, Michaeli J, Assi S, et al. Phenotypic diversity and plasticity in circulating neutrophil subpopulations in cancer. Cell Rep. 2015;10(4):562-73.

100. Tay SH, Celhar T, Fairhurst AM. Low-density neutrophils in systemic lupus erythematosus. Arthritis Rheumatol. 2020;72(10):1587-95.

101. Ng LG, Ostuni R, Hidalgo A. Heterogeneity of neutrophils. Nat Rev Immunol. 2019;19(4):255-65.

102. Hassani M, Hellebrekers $P$, Chen $N$, et al. On the origin of low-density neutrophils. J Leukoc Biol. 2020;107(5):809-18.

103. Papayannopoulos V, Metzler KD, Hakkim A, Zychlinsky A. Neutrophil elastase and myeloperoxidase regulate the formation of neutrophil extracellular traps. J Cell Biol. 2010;191(3):677-91.

104. Cundall M, Sun Y, Miranda C, et al. Neutrophil-derived matrix metalloproteinase-9 is increased in severe asthma and poorly inhibited by glucocorticoids. J Allergy Clin Immunol. 2003;112(6):1064-71.

105. Fujishima S, Hoffman AR, Vu T, et al. Regulation of neutrophil interleukin 8 gene expression and protein secretion by LPS, TNF-alpha, and IL-1 beta. J Cell Physiol. 1993;154(3):478-85.

106. Ding Q, Sun S, Zhang Y, et al. Serum IL-8 and VEGFA are Two Promising Diagnostic Biomarkers of Asthma-COPD Overlap Syndrome. Int J Chron Obstruct Pulmon Dis. 2020;15:357-65.

107. Dimitrova D, Youroukova V, Ivanova-Todorova E, TumangelovaYuzeir K, Velikova T. Serum levels of IL-5, IL-6, IL-8, IL-13 and IL-17A in pre-defined groups of adult patients with moderate and severe bronchial asthma. Respir Med. 2019;154:144-54.

108. Jiang XG, Yang XD, Lv Z, Zhuang PH. Elevated serum levels of TNF-alpha, IL-8, and ECP can be involved in the development and progression of bronchial asthma. J Asthma. 2018;55(2):111-8.

109. Norzila MZ, Fakes K, Henry RL, Simpson J, Gibson PG. Interleukin- 8 secretion and neutrophil recruitment accompanies induced sputum eosinophil activation in children with acute asthma. Am J Respir Crit Care Med. 2000;161(3 Pt 1):769-74.

110. Hamilton JA. GM-CSF in inflammation. J Exp Med. 2020. https://doi.org/10.1084/jem.20190945.

111. Mattoli S, Marini M, Fasoli A. Expression of the potent inflammatory cytokines, GM-CSF, IL6, and IL8, in bronchial epithelial cells of asthmatic patients. Chest. 1992;101(3 Suppl):27S-29S.

112. Saha S, Doe C, Mistry V, et al. Granulocyte-macrophage colonystimulating factor expression in induced sputum and bronchial mucosa in asthma and COPD. Thorax. 2009;64(8):671-6.

113. Woolley KL, Adelroth E, Woolley MJ, et al. Granulocyte-macrophage colony-stimulating factor, eosinophils and eosinophil cationic protein in subjects with and without mild, stable, atopic asthma. Eur Respir J. 1994;7(9):1576-84.

114. Detoraki A, Granata F, Staibano S, et al. Angiogenesis and lymphangiogenesis in bronchial asthma. Allergy. 2010;65(8):946-58.

115. Duah E, Teegala LR, Kondeti V, et al. Cysteinyl leukotriene 2 receptor promotes endothelial permeability, tumor angiogenesis, and metastasis. Proc Natl Acad Sci U S A. 2019;116(1):199-204.

116. Detoraki A, Staiano RI, Granata F, et al. Vascular endothelial growth factors synthesized by human lung mast cells exert angiogenic effects. J Allergy Clin Immunol. 2009;123(5):1142-9.

117. Marone G, Rossi FW, Pecoraro A, et al. HIV gp120 induces the release of proinflammatory, angiogenic, and lymphangiogenic factors from human lung mast cells. Vaccines (Basel). 2020;8(2):208.

118. Staiano RI, Loffredo S, Borriello F, et al. Human lung-resident macrophages express $\mathrm{CB} 1$ and $\mathrm{CB} 2$ receptors whose activation inhibits the release of angiogenic and lymphangiogenic factors. J Leukoc Biol. 2016;99(4):531-40.

119. de Paulis A, Prevete N, Fiorentino I, et al. Expression and functions of the vascular endothelial growth factors and their receptors in human basophils. J Immunol. 2006;177(10):7322-31.

120. Nissim Ben Efraim, AH, Levi Schaffer F, Roles of eosinophils in the modulation of angiogenesis. Chem Immunol Allergy. 2014; 99: 138-54.

121. Lee KS, Kim SR, Park SJ, et al. Hydrogen peroxide induces vascular permeability via regulation of vascular endothelial growth factor. Am J Respir Cell Mol Biol. 2006;35(2):190-7.

122. Reber LL, Gillis CM, Starkl P, et al. Neutrophil myeloperoxidase diminishes the toxic effects and mortality induced by lipopolysaccharide. J Exp Med. 2017;214(5):1249-58.

123. Takei H, Araki A, Watanabe H, Ichinose A, Sendo F. Rapid killing of human neutrophils by the potent activator phorbol 12-myristate 13-acetate (PMA) accompanied by changes different from typical apoptosis or necrosis. J Leukoc Biol. 1996;59(2):229-40.

124. Yipp BG, Kubes P. NETosis: how vital is it? Blood. 2013;122(16):2784-94.

125. Xiao F, Jiang Y, Wang X, et al. NETosis may play a role in the pathogenesis of Hashimoto's thyroiditis. Int J Clin Exp Pathol. 2018;11(2):537-47.

126. Wright TK, Gibson PG, Simpson JL, et al. Neutrophil extracellular traps are associated with inflammation in chronic airway disease. Respirology. 2016;21(3):467-75.

127. Krishnamoorthy N, Douda DN, Bruggemann TR, et al. Neutrophil cytoplasts induce TH17 differentiation and skew inflammation toward neutrophilia in severe asthma. Sci Immunol. 2018;3(26):eaao4747.

128. Yousefi S, Simon D, Stojkov D, et al. In vivo evidence for extracellular DNA trap formation. Cell Death Dis. 2020;11(4):300. 
129. Hayden H, Ibrahim N, Klopf J, et al. ELISA detection of MPODNA complexes in human plasma is error-prone and yields limited information on neutrophil extracellular traps formed in vivo. PLoS One. 2021;16(4):e0250265.

130. Granger V, Taille C, Roach D, et al. Circulating neutrophil and eosinophil extracellular traps are markers of severe asthma. Allergy. 2020;75(3):699-702.

131. Robledo-Avila FH, Ruiz-Rosado JD, Brockman KL, et al. Dysregulated calcium homeostasis in cystic fibrosis neutrophils leads to deficient antimicrobial responses. J Immunol. 2018;201(7):2016-27.

132. Yipp BG, Petri B, Salina D, et al. Infection-induced NETosis is a dynamic process involving neutrophil multitasking in vivo. Nat Med. 2012;18(9):1386-93.

133. Zhang Y, Chandra V, Riquelme Sanchez E, et al. Interleukin17-induced neutrophil extracellular traps mediate resistance to checkpoint blockade in pancreatic cancer. J Exp Med. 2020. https://doi.org/10.1084/jem.20190354.

134. Jin R, Xu J, Gao Q, et al. IL-33-induced neutrophil extracellular traps degrade fibronectin in a murine model of bronchopulmonary dysplasia. Cell Death Discov. 2020;6:33.

135. Wang X, Li X, Chen L, et al. Interleukin-33 facilitates cutaneous defense against Staphylococcus aureus by promoting the development of neutrophil extracellular trap. Int Immunopharmacol. 2020;81:106256.

136. Nie M, Yang L, Bi X, et al. Neutrophil Extracellular Traps Induced by IL8 Promote Diffuse Large B-cell Lymphoma Progression via the TLR9 Signaling. Clin Cancer Res. 2019;25(6):1867-79.

137. An Z, Li J, Yu J, et al. Neutrophil extracellular traps induced by IL-8 aggravate atherosclerosis via activation NF-kappaB signaling in macrophages. Cell Cycle. 2019;18(21):2928-38.

138. Alfaro C, Teijeira A, Onate C, et al. Tumor-produced interleukin-8 attracts human myeloid-derived suppressor cells and elicits extrusion of neutrophil extracellular traps (NETs). Clin Cancer Res. 2016;22(15):3924-36.
139. Teijeira A, Garasa S, Gato M, et al. CXCR1 and CXCR2 chemokine receptor agonists produced by tumors induce neutrophil extracellular traps that interfere with immune cytotoxicity. Immunity. 2020;52(5):856-71.

140. Guglietta S, Chiavelli A, Zagato E, et al. Coagulation induced by C3aR-dependent NETosis drives protumorigenic neutrophils during small intestinal tumorigenesis. Nat Commun. 2016;7:11037.

141. Meher AK, Spinosa M, Davis JP, et al. Novel Role of IL (Interleukin)-1beta in Neutrophil Extracellular Trap Formation and Abdominal Aortic Aneurysms. Arterioscler Thromb Vasc Biol. 2018;38(4):843-53.

142. Marino F, Scanzano A, Pulze L, et al. beta2 -Adrenoceptors inhibit neutrophil extracellular traps in human polymorphonuclear leukocytes. J Leukoc Biol. 2018;104(3):603-14.

143. Tcherniuk S, Cenac N, Comte M, et al. Formyl peptide receptor 2 plays a deleterious role during influenza A virus infections. $\mathbf{J}$ Infect Dis. 2016;214(2):237-47.

144. Kalchiem-Dekel O, Yao X, Levine SJ. Meeting the challenge of identifying new treatments for type 2-Low neutrophilic asthma. Chest. 2020;157(1):26-33.

145. Fahy JV. Type 2 inflammation in asthma-present in most, absent in many. Nat Rev Immunol. 2015;15(1):57-65.

146. Peters MC, Wenzel SE. Intersection of biology and therapeutics: type 2 targeted therapeutics for adult asthma. Lancet. 2020;395(10221):371-83.

147. Sze E, Bhalla A, Nair P. Mechanisms and therapeutic strategies for non-T2 asthma. Allergy. 2020;75(2):311-25.

Publisher's Note Springer Nature remains neutral with regard to jurisdictional claims in published maps and institutional affiliations. 